\title{
Efectos de la inducción emocional usando realidad virtual en la percepción de bienestar en personas vulnerables a padecer ansiedad y depresión
}

\author{
CRISTINA FERNÁNDEZ RAMOS \\ al202185@uji.es \\ Cristina Botella Arbona \\ botella@uji.es \\ BERENICE SERRANo ZÁRATE \\ bserrano@uji.es
}

\section{Resumen}

Introducción: La incidencia de trastornos como la depresión o la ansiedad es muy alta en todo el mundo. Se ha observado que las emociones positivas pueden funcionar como factor protector contra estos trastornos y que la tecnología como la realidad virtual (RV) podría ser de utilidad como procedimiento de inducción emocional (PIE). Por ello, la inducción de emociones positivas podría jugar un papel fundamental en la prevención de estas psicopatologías. El objetivo del presente trabajo fue someter a prueba en un estudio piloto si la inducción de emociones positivas mediante RV podía modificar la percepción subjetiva de bienestar. Método: El estudio se realizó con 10 voluntarios vulnerables a padecer ansiedad o depresión que no habían recibido diagnóstico para ninguno de estos dos trastornos. Se llevó a cabo la inducción de relajación o alegría mediante PIE con apoyo de RV. Resultados: Los resultados muestran una tendencia a reducir la intensidad del estado emocional negativo y a incrementar la intensidad de las emociones positivas. Respecto a la percepción de bienestar, se identificaron cambios positivos después de la inducción emocional. Conclusión: Los resultados obtenidos no son concluyentes; sin embargo, es importante considerar que, debido a la importancia de la prevención de trastornos como la ansiedad y la depresión, sería recomendable seguir investigando los potenciales efectos de la inducción de emociones positivas sobre la percepción de bienestar y su vínculo con la salud mental, así como su potencial utilidad en la prevención de la psicopatología.

Palabras clave: inducción emocional, realidad virtual, bienestar, ansiedad, depresión.

\section{Abstract}

Introduction: The incidence of mental disorders like depression or anxiety is very high worldwide. Positive emotions have been found to work as a protective factor against these disorders, and that technology like virtual reality (VR) can be useful 
as a mood induction procedure (MIP). Therefore inducing these emotions could play a fundamental role in the prevention of these psychopathologies. The objective of this paper was to run a pilot study to test if induction of positive emotions using VR can modify the subjective perception of well-being. Method: The study was conducted in a sample of 10 volunteers who were vulnerable to suffer anxiety and/or depression, and who had not been diagnosed for either of these two disorders. Relaxation or joy was induced using different MIPS supported by VR. Results: The results showed the following tendency: intensity of negative emotions diminished and intensity of positive emotions increased. Regarding the perception of well-being, positive changes were found after mood induction. Conclusion: Although the results are not conclusive, it is important to consider that given the importance of preventing disorders like anxiety and depression, continuing to research into the potential effects of inducing positive emotions on the perception of well-being, and its link to mental health, as well as its potential usefulness in psychopathology prevention, is recommended.

Keywords: mood induction, virtual reality, well-being, anxiety, depression.

\section{Introducción}

Las emociones positivas, además de hacernos sentir bien (Livingstone y Srivatava, 2012), pueden ser capaces de deshacer los efectos psicológicos y fisiológicos producidos por las emociones negativas, lo cual permite una recuperación más óptima y rápida frente a experiencias estresantes (Folkman y Moskowitz, 2000). Según la teoría de ampliación y construcción de emociones positivas (Fredrickson, 1998), este tipo de emociones ayudarían a ampliar la atención, la cognición y la acción, lo cual aportaría a la persona la construcción de recursos físicos, cognitivos y sociales a largo plazo para poder afrontar situaciones difíciles o problemáticas futuras. De esta forma, las emociones positivas amplían el alcance de nuestros pensamientos y acciones, así como incrementan los repertorios de los mismos, facilitando, de este modo, el desarrollo de nuevas formas de afrontamiento.

En la última década, se ha explorado el uso de las tecnologías de la información y comunicación (TIC) como medio facilitador para inducir emociones. Los estudios se han centrado principalmente en el uso de la realidad virtual (RV), la cual ha demostrado su potencial utilidad para modificar las emociones (Botella y cols., 2009) y su capacidad para generar altos niveles de sentido de presencia (Baños y cols., 2004). Así mismo, ningún estudio ha reportado efectos secundarios por el uso de la RV en la inducción de emociones. Uno de los primeros estudios realizados por Baños y cols. (2004) sometió a prueba un procedimiento de inducción emocional (PIE) usando RV denominado «Parques». Este PIE con RV suponía un ambiente virtual basado en un entorno neutral que cambia progresivamente dependiendo de la emoción que se pretende evocar. Es importante añadir que en este sistema de RV se integraron diferentes PIE clásicos para inducir alegría, tristeza, ansiedad y relajación. Estos autores encontraron que este PIE con RV era capaz de producir un cambio en el estado emocional de los participantes. De esta forma, se observó que mediante estos PIE era posible inducir emociones específicas en los usuarios. Estos resultados son de gran importancia ya que revelan la utilidad de la RV como un dispositivo que se puede emplear en la inducción de diferentes emociones. En otro estudio realizado por Baños y cols. (2006) se utilizó el mismo PIE pero con la diferencia de que 
solo se llevó a cabo la inducción de alegría y tristeza en población universitaria. Se encontró que este PIE no solo fue eficaz para inducir la emoción objetivo, sino que también fue capaz de cambiar el estado de ánimo inducido a la emoción opuesta. En la misma línea, Baños y cols. (2013) realizaron un estudio para explorar la factibilidad de una intervención psicológica usando este PIE con RV, centrado en la inducción de emociones positivas en pacientes de oncología hospitalizados, quienes se encontraban en estadios muy avanzados de la enfermedad. Los resultados obtenidos fueron positivos. Se observó que, después de cada una de las sesiones en las que se aplicó el PIE con RV, hubo un cambio en el estado de ánimo de los participantes, con un incremento en la intensidad de los estados emocionales positivos y un decremento en la intensidad de los estados emocionales negativos.

Por otra parte, varios estudios han probado que la promoción de la salud mental así como la utilización de intervenciones de carácter preventivo ayudan a reducir el riesgo de padecer un trastorno mental en el futuro (Barry, Clark y Pettersen, 2015) y, tomando en cuenta que se ha probado la eficacia de la inducción de emociones positivas con RV, consideramos que sería beneficioso estudiar su utilidad como intervención preventiva en el desarrollo de diferentes trastornos psicológicos, como son la depresión y la ansiedad, los cuales tienen una incidencia muy alta en la actualidad (Organización Mundial de la Salud, 2011). Además, ya que parece que las emociones positivas pueden reducir las consecuencias derivadas de las emociones negativas (Wood y Tarrier, 2010), estas podrían jugar un papel fundamental en la prevención de diferentes trastornos debido a que pueden funcionar como factor protector contra el estrés y la depresión (Santos y cols., 2013).

De este modo, este estudio tiene como objetivo someter a prueba si la inducción de emociones positivas mediante RV modifica la percepción subjetiva de bienestar en personas vulnerables a padecer ansiedad o depresión; así mismo, se evalúan otras variables vinculadas al uso de la RV.

Las hipótesis planteadas son las siguientes:

- H1: Después de la inducción emocional con RV, se incrementará el nivel de alegría y relajación y disminuirá la intensidad de la tristeza y ansiedad en los participantes.

- H2: La percepción subjetiva de bienestar se incrementará en los participantes tras la inducción de emociones positivas.

- H3: El sentido de presencia será elevado en los participantes.

- H4: No se identificarán efectos secundarios al uso de la RV en los participantes.

\section{Método}

\section{Participantes}

La muestra está compuesta por un total de 10 voluntarios, de los cuales 8 son mujeres. El rango de edad oscilaba entre los 18 y los 54 años (media $=28,3$; DS $=13,5$ ). De las 10 personas voluntarias, el $40 \%$ tenía un nivel de estudios superiores, el $30 \%$ un nivel de estudios medio y el $10 \%$ un nivel de estudios básico. Ninguno de los participantes había recibido un diagnóstico de ansiedad o depresión; sin embargo, el $50 \%$ tenía un familiar o familiares diagnosticados de algún problema de ansiedad y el $60 \%$ tenía un familiar o familiares con diagnóstico de depresión. Así mismo, ninguno de los participantes tomaba medicación.

Para reclutar la muestra se elaboraron carteles informativos y se colocaron en lugares estratégicos de la Universitat Jaume I. Antes del pase experimental, todos los participantes cumplimentaron una serie de cuestionarios (BDI-II, ASI-III y SCID-II escala de depresión) a través 
de SurveyMonkey, con el objetivo de identificar si cumplían el criterio de inclusión (tener entre 18 y 65 años) y el criterio de exclusión (una puntuación en el BDI-II superior a 19) y de evaluar qué condición experimental les sería asignada en base a los síntomas identificados. Los participantes fueron asignados a una de las dos condiciones experimentales: 1) inducción de alegría o 2) relajación, en base al nivel de malestar e interferencia de los síntomas reportados en los cuestionarios antes citados.

\section{Medidas}

Se administraron los siguientes instrumentos de medida:

- Inventario de depresión de Beck (BDI-II; Beck, Ward, Mendelson, Mock y Erbaugh, 1961). Se utilizó la versión adaptada para población española de Sanz y Vázquez (1998). Se trata de una medida de autoinforme que consta de 21 ítems dirigidos a evaluar la sintomatología propia de los trastornos depresivos.

- Inventario de ansiedad estado-rasgo (STAl; Spielberger, Gorsuch y Lushene, 1970). Se utilizó la traducción al castellano de TEA ediciones, de la escala para evaluar la ansiedad estado (STAI-E), tanto antes como después de la realización de la tarea experimental.

- Entrevista clínica estructurada para los trastornos de personalidad del eje II del DSM-IV (SCID-II; First, Gibbon, Spitzer, Williams y Benjamín, 1999). Entrevista semiestructurada diseñada para realizar diagnósticos de personalidad en base a los criterios del DSM. Para este estudio solo fue utilizada la escala depresión.

- Índice de sensibilidad a la ansiedad (ASI-III; Peterson y Reiss, 1992). Se utilizó la validación española de Sandín, Valiente, Chorot y Santed (2007). Consta de un total de 16 ítems, a los que los participantes contestan según una escala tipo Likert de cuatro puntos.

- Escala de afecto positivo y negativo (PANAS; Watson, Clark y Tellegen, 1988). Se utilizó la versión traducida al castellano por Sandín y cols., 1999). La escala consta de una lista de 10 ítems que miden emociones positivas y 10 que miden emociones negativas.

- Índice de calidad de vida (QLI; Mezzich, Cohen y Ruiperez, 1999). Índice compuesto por 10 ítems, medidos en una escala 1-10, que representan diferentes constructos incluidos en la calidad de vida: bienestar físico, bienestar psicológico-emocional, autocuidado y funcionamiento independiente, funcionamiento ocupacional, funcionamiento interpersonal, apoyo socioemocional, apoyo comunitario y de servicios, plenitud personal, plenitud espiritual y percepción global de calidad de vida.

- Escalas analógico visuales (VAS; Gross y Levenson, 1985). Las escalas VAS son instrumentos en los que se pide a la persona que valore, en este caso, el grado en el que siente en ese momento una emoción determinada. Para este estudio se utilizó una versión adaptada por Baños y cols. (2004) en la cual solo se mide alegría, ansiedad, tristeza y relajación en una escala 0-10.

- Cuestionario de cibermareos (Kennedy, Lane, Berbaum y Lilienthal, 1993). Se utilizó la validación de Bouchard, Robillard, Renaud y Bernier (2011) traducida para este estudio. Este cuestionario identifica sintomatología física producida como efecto negativo de la inmersión en RV. En ella se diferencian dos factores: síntomas de náuseas y síntomas oculomotores. 
- Cuestionario de presencia y juicio de realidad (CPYJR; Baños y cols., 2000). Fue diseñado para evaluar distintos componentes del sentido de presencia. El cuestionario está integrado por cinco factores medidos en una escala 0-10: implicación emocional, juicio de realidad y presencia, influencia de las variables formales en el juicio de realidad y sentido de presencia y satisfacción con la experiencia.

- Opinión de la experiencia emocional en realidad virtual. Esta escala de elaboración específica para este estudio fue creada para identificar el grado de utilidad y de satisfacción, medidos en una escala 0-10, que valoraban los participantes acerca de la experiencia de inducción emocional con el ambiente virtual.

\section{Ambiente de realidad virtual}

Se trata de un parque (figura 1) en el que cambian algunos elementos (por ejemplo, colores, intensidad de la luz, etc.) dependiendo del estado de ánimo que se pretenda inducir. En este ambiente se encuentran distintos PIE integrados en el entorno virtual. Este sistema de RV cuenta con los siguientes PIE: frases autorreferentes de Velten, música y sonidos, sistema internacional de imágenes afectivas y recuerdo autobiográfico. La persona es guiada en todo momento por una narrativa la cual indica qué tareas de inducción emocional tiene que ir realizando.

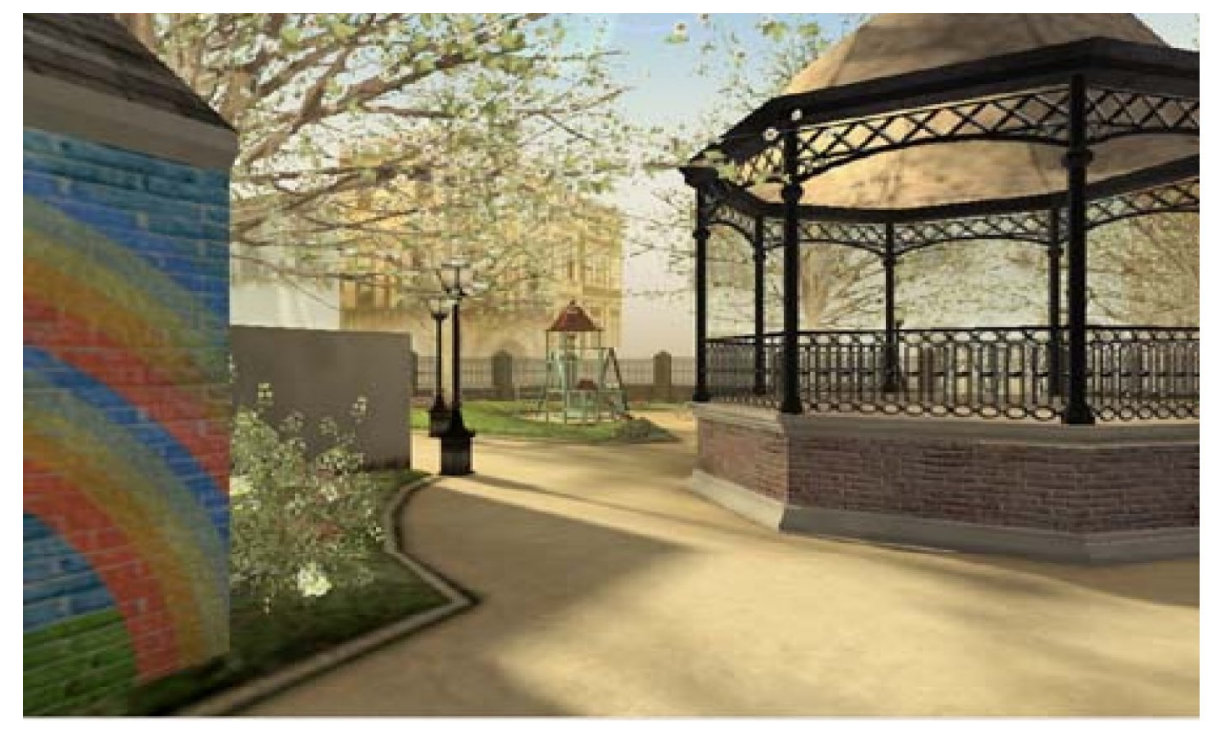

Figura 1. Parque para inducir alegría

\section{Procedimiento}

En primer lugar, las personas voluntarias rellenaron los siguientes cuestionarios: BDI-II, ASI-III y la escala de depresión de la SCID-II a través de SurveyMonkey con el objetivo de identificar si cumplían el criterio de exclusión y de evaluar qué condición experimental (alegría o relajación) les sería asignada en base a los síntomas identificados. Al llamamiento respondieron 15 participantes de los cuales fueron excluidos 5 por presentar una puntuación en el BDI-II 
superior a 19. Para este estudio se consideró una muestra que mostrara síntomas subclínicos de ansiedad y depresión en niveles leves y moderados. La asignación a cada condición experimental se realizó en base a los niveles de malestar, evitación e interferencia de los síntomas. Si los síntomas predominantes eran de ansiedad, los participantes eran asignados a la condición relajación; si los síntomas predominantes eran de depresión eran asignados a la condición alegría. Fueron asignados cinco participantes a cada una de las condiciones experimentales.

Los participantes rellenaron un consentimiento informado y una hoja de datos sociodemográficos desarrollada específicamente para este estudio. Posteriormente, realizaron una evaluación previa a la inducción emocional en donde rellenaron los siguientes cuestionarios anteriormente citados: QLI, STAI-E, BDI-II, PANAS, VAS y cuestionario de cibermareos. A continuación, los participantes llevaron a cabo un entrenamiento de cinco minutos aproximadamente con el objetivo de aprender cómo utilizar el entorno de RV. Posteriormente, se les dejaba solos en la sala y entraban en el parque virtual asignado (parque alegre o parque relajación); la duración de la inducción emocional fue de 20 minutos aproximadamente y se realizó en una sola sesión. La experiencia con el parque en RV comienza con una narrativa en la cual la voz de una mujer les introduce y les guía por las diferentes tareas de inducción emocional a realizar en el parque. Después, los participantes pueden pasear por el parque hasta llegar a un quiosco, en el cual se les indican las primeras tareas a realizar: ordenar una serie de frases autorreferentes y seleccionar para estas frases una imagen procedente del sistema de imágenes afectivas; en seguida, los participantes pueden seguir paseando por el parque mientras escuchan en todo momento una música acorde con el estado emocional que se pretende inducir; posteriormente, realizan la última tarea que consiste en evocar un recuerdo autobiográfico. Al finalizar la experimentación, los participantes rellenaron los siguientes cuestionarios: QLI, STAI-E, BDI-II, PANAS, VAS, cuestionario de cibermareos, CPJR y cuestionario de opinión de la experiencia emocional en realidad virtual.

\section{Resultados}

Para someter a prueba si la inducción de emociones positivas mediante RV puede modificar la percepción subjetiva de bienestar en personas vulnerables a padecer ansiedad y depresión, se llevó a cabo un análisis de comparación de medias mediante la utilización de la prueba $t$ (tabla 1). La comparación de medias en las medidas emocionales muestra diferencias significativas en la condición relajación (figura 2): el nivel de relajación fue significativamente mayor después de la inducción emocional $(t(4)=-3,47, p=0,025)$. Sin embargo, no se muestran diferencias significativas para la condición alegría.

También se identificaron cambios significativos en otras de las variables evaluadas; ambas condiciones mostraron niveles significativamente menores después de la inducción emocional en el STAI-estado: condición alegría $(t(4)=10,06, p=0,001)$, condición relajación $(t(4)=4,18, p=0,014)$; solo fueron identificados cambios significativos en el PANAS para las emociones negativas en la condición alegría $(t(4)=5,65, p=0,005)$, siendo estas menores después de la inducción emocional. 
Tabla 1

Comparación de medias de las variables estudiadas

\begin{tabular}{|c|c|c|c|c|}
\hline & \multicolumn{2}{|c|}{ Condición alegría (n=5) } & \multicolumn{2}{|c|}{ Condición relajación $(n=5)$} \\
\hline & PRE-TEST & POST-TEST & PRE-TEST & POST-TEST \\
\hline BDI-II & $\begin{array}{c}\bar{X}=13,80 \\
(D S=4,65)\end{array}$ & $\bar{X}=12(D S=6,36)$ & $\bar{X}=10(D S=10,18)$ & $\begin{array}{c}\bar{X}=7,20 \\
(D S=10,18)\end{array}$ \\
\hline StAI-E & $\begin{array}{c}\bar{X}=27,80 \\
(D S=6,53)\end{array}$ & $\begin{array}{c}\bar{X}=18,80 \\
(D S=6,79)\end{array}$ & $\begin{array}{c}\bar{X}=20,40 \\
(D S=13,86)\end{array}$ & $\begin{array}{c}\bar{X}=6,20 \\
(\mathrm{DS}=6,87)\end{array}$ \\
\hline PANAS POSITIVO & $\begin{array}{c}\bar{X}=31,80 \\
(D S=6,61)\end{array}$ & $\begin{array}{c}\bar{X}=29,20 \\
(D S=6,76)\end{array}$ & $\bar{X}=31(D S=3,08)$ & $\begin{array}{c}\bar{X}=37,20 \\
(D S=8,46)\end{array}$ \\
\hline PANAS NEGATIVO & $\bar{X}=23(D S=6,67)$ & $\bar{X}=19(D S=6,16)$ & $\begin{array}{c}\bar{X}=20,40 \\
(D S=3,91)\end{array}$ & $\bar{X}=15(D S=7,03)$ \\
\hline VAS- TRISTEZA & $\begin{array}{c}\bar{X}=4,40 \\
(D S=2,70)\end{array}$ & $\bar{X}=2(D S=2,34)$ & $\bar{X}=1,60(D S=0,89)$ & $\begin{array}{c}\bar{X}=1,20 \\
(D S=2,16)\end{array}$ \\
\hline VAS-ALEGRÍA & $\begin{array}{c}\bar{X}=6,60 \\
(D S=1,14)\end{array}$ & $\begin{array}{c}\bar{X}=7,20 \\
(D S=1,64)\end{array}$ & $\bar{X}=6,80(D S=1,30)$ & $\begin{array}{c}\bar{X}=7,20 \\
(D S=1,78)\end{array}$ \\
\hline VAS-ANSIEDAD & $\begin{array}{c}\bar{X}=3,20 \\
(D S=2,58)\end{array}$ & $\bar{X}=3(D S=2,82)$ & $\bar{X}=2,40(D S=2,79)$ & $\begin{array}{c}\bar{X}=0,40 \\
(D S=0,89)\end{array}$ \\
\hline VAS-RELAJACIÓN & $\begin{array}{c}\bar{X}=5,80 \\
(D S=3,03)\end{array}$ & $\begin{array}{c}\bar{X}=6,60 \\
(D S=2,51)\end{array}$ & $\bar{X}=6,20(D S=1,92)$ & $\begin{array}{c}\bar{X}=8,80 \\
(D S=1,30)\end{array}$ \\
\hline $\begin{array}{l}\text { CIBERMAREOS- } \\
\text { NAUSEAS }\end{array}$ & $\begin{array}{c}\bar{X}=1,60 \\
(D S=1,14)\end{array}$ & $\begin{array}{c}\bar{X}=2,40 \\
(D S=3,20)\end{array}$ & $\bar{X}=0,80(D S=1,30)$ & $\bar{X}=1(D S=1,41)$ \\
\hline CIBERMAREOS-OCULAR & $\begin{array}{c}\bar{X}=1,80 \\
(D S=1,30)\end{array}$ & $\begin{array}{c}\bar{X}=1,80 \\
(D S=2,38)\end{array}$ & $\bar{X}=2,20(D S=2,28)$ & $\begin{array}{c}\bar{X}=0,60 \\
(D S=0,89)\end{array}$ \\
\hline QLI-FÍsıCO & $\begin{array}{c}\bar{X}=7,40 \\
(D S=1,51)\end{array}$ & $\begin{array}{c}\bar{X}=7,80 \\
(\mathrm{DS}=1,48)\end{array}$ & $\bar{X}=6,80(\mathrm{DS}=1,92)$ & $\begin{array}{c}\bar{X}=8,40 \\
(D S=1,51)\end{array}$ \\
\hline QLI-PSICOLÓGICO & $\begin{array}{c}\bar{X}=7,60 \\
(D S=1,51)\end{array}$ & $\begin{array}{c}\bar{X}=8,20 \\
(D S=1,09)\end{array}$ & $\bar{X}=6,80(D S=1,30)$ & $\begin{array}{c}\bar{X}=8,40 \\
(\mathrm{DS}=0,89)\end{array}$ \\
\hline QLI-AUTOCUIDADO & $\begin{array}{c}\bar{X}=7,80 \\
(D S=1,64)\end{array}$ & $\begin{array}{c}\bar{X}=7,80 \\
(D S=1,64)\end{array}$ & $\bar{X}=7,80(\mathrm{DS}=1,92)$ & $\begin{array}{c}\bar{X}=8,40 \\
(D S=1,81)\end{array}$ \\
\hline QLI-OCUPACIONAL & $\begin{array}{c}\bar{X}=7,40 \\
(\mathrm{DS}=2,70)\end{array}$ & $\begin{array}{c}\bar{X}=7,60 \\
(D S=2,30)\end{array}$ & $\bar{X}=6,80(D S=1,64)$ & $\begin{array}{c}\bar{X}=8,40 \\
(D S=1,14)\end{array}$ \\
\hline QLI-INTERPERSONAL & $\begin{array}{c}\bar{X}=8,40 \\
(D S=1,14)\end{array}$ & $\begin{array}{c}\bar{X}=8,60 \\
(\mathrm{DS}=0,89)\end{array}$ & $\bar{X}=8,20(D S=1,64)$ & $\begin{array}{c}\bar{X}=8,80 \\
(\mathrm{DS}=1,30)\end{array}$ \\
\hline QLI-SOCIAL & $\begin{array}{c}\bar{X}=8,60 \\
(D S=1,14)\end{array}$ & $\begin{array}{c}\bar{X}=8,80 \\
(D S=0,83)\end{array}$ & $\bar{X}=7,20(D S=2,16)$ & $\begin{array}{c}\bar{X}=8,40 \\
(\mathrm{DS}=2,07)\end{array}$ \\
\hline QLI-COMUNITARIO & $\begin{array}{c}\bar{X}=8,40 \\
(D S=1,14)\end{array}$ & $\begin{array}{c}\bar{X}=8,20 \\
(D S=1,30)\end{array}$ & $\bar{X}=6,40(D S=2,30)$ & $\begin{array}{c}\bar{X}=6,40 \\
(\mathrm{DS}=2,51)\end{array}$ \\
\hline QLI-PERSONAL & $\bar{X}=7,2(D S=2,68)$ & $\begin{array}{c}\bar{X}=7,60 \\
(D S=2,30)\end{array}$ & $\bar{X}=7(D S=1,87)$ & $\bar{X}=8(D S=1,41)$ \\
\hline QLI-ESPIRITUAL & $\bar{X}=7,2(\mathrm{DS}=2,04)$ & $\begin{array}{c}\bar{X}=7,20 \\
(D S=2,04)\end{array}$ & $\bar{X}=7(D S=1,87)$ & $\begin{array}{c}\bar{X}=8,20 \\
(D S=1,78)\end{array}$ \\
\hline QLI-GLOBAL & $\bar{X}=8(D S=1,22)$ & $\begin{array}{c}\bar{X}=7,80 \\
(D S=1,09)\end{array}$ & $\bar{X}=7(D S=1,87)$ & $\begin{array}{c}\bar{X}=8,20 \\
(D S=1,09)\end{array}$ \\
\hline
\end{tabular}




\section{VAS Condición Relajación}

$\square$ PRE-TEST $\square$ POST-TEST

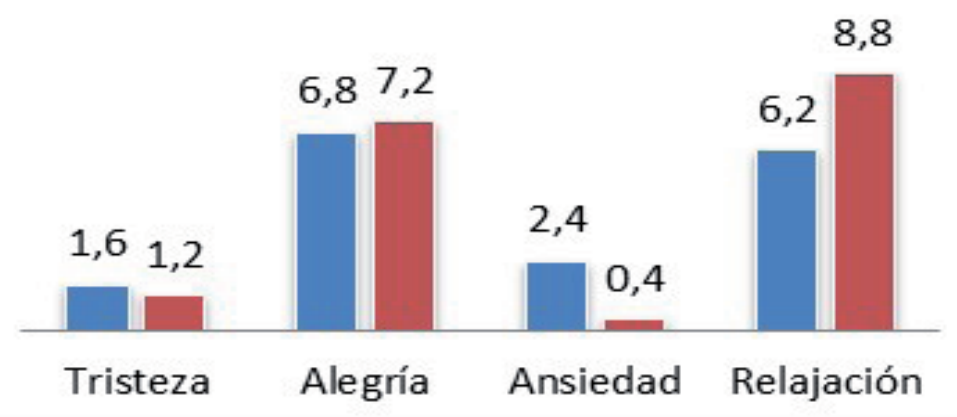

Figura 2. Variables emocionales en el grupo con vulnerabilidad a la ansiedad

En lo que respecta a las variables de calidad de vida, se observan diferencias significativas en algunas de las variables en la condición relajación (figura 3): la percepción subjetiva de calidad de vida se vio significativamente incrementada tras la inducción emocional en las variables física $(t=-4(4), p=0,016)$, psicológica $(t=-4(4), p=0,016)$, personal $(t=-3,16(4)$, $p=0,034)$ y global $(t=-3,20(4), p=0,033)$. Sin embargo, no se aprecian diferencias significativas en la condición alegría.

\section{QLI condición relajación}

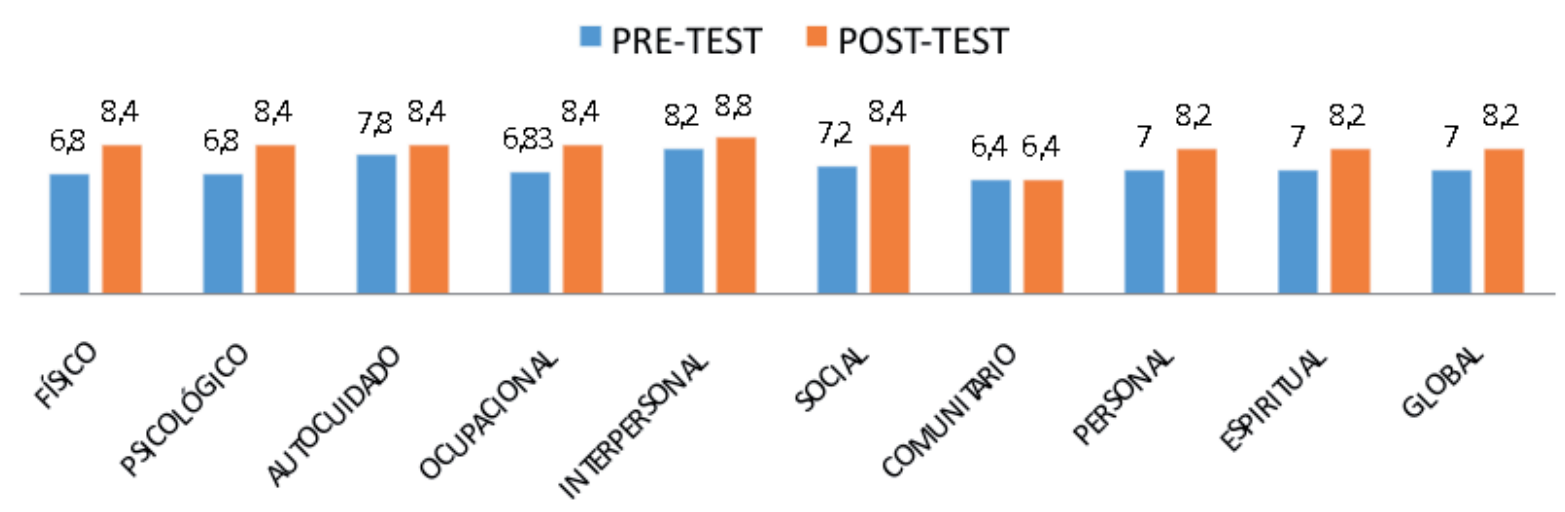

Figura 3. Variables de calidad de vida en la condición de relajación

En relación al sentido de presencia en cada uno de los factores evaluados, se identificaron altos niveles en la condición relajación, pero niveles moderados en la condición alegría, tal como puede observarse en la tabla 2. 
Tabla 2

Niveles de presencia durante la inducción emocional con RV

\begin{tabular}{lcc}
\hline & $\begin{array}{c}\text { Condición alegría } \\
\text { Media (DT) }\end{array}$ & $\begin{array}{c}\text { Condición relajación } \\
\text { (Media (DT) }\end{array}$ \\
\hline Implicación emocional & $5,08(1,50)$ & $7,80(1,66)$ \\
Juicio de realidad y presencia & $5,60(1,63)$ & $7,62(2,22)$ \\
Interacción y correspondencia externa & $6,70(2,38)$ & $8(1,21)$ \\
Influencia de las variables formales & $6,51(1,21)$ & $8,14(1,95)$ \\
Satisfacción con la experiencia & $7,60(1,40)$ & $8,90(1,20)$ \\
\hline
\end{tabular}

Respecto a los efectos secundarios del uso de la RV, no se identificaron cambios significativos después de la inducción emocional en ninguna de las dos condiciones experimentales.

También se evaluó la opinión de los participantes acerca de la experiencia de inducción emocional en RV. En la tabla 3 se muestran las puntuaciones medias en cada una de las condiciones. Se puede observar que hubo una buena aceptación del PIE apoyado por RV. Así mismo, en la condición de alegría, el $40 \%$ de los participantes refirieron que sí utilizaría un sistema de RV en casa para mejorar sus emociones con una frecuencia de dos a tres veces por semana; el $20 \%$ lo utilizaría de cuatro a cinco veces a la semana, otro $20 \%$ una vez a la semana y solo un $20 \%$ refirió que no lo utilizaría. En la condición de relajación, todos los participantes refirieron que lo utilizarían; un 40 \% lo utilizaría de cuatro a cinco veces a la semana, otro $40 \%$ lo usaría de dos a tres veces por semana y un $20 \%$ una vez a la semana.

Respecto a en qué tipo de dispositivo les gustaría poder utilizarlo, en la condición de alegría al $50 \%$ les gustaría disponer de él en un TV, el $25 \%$ en un ordenador y el $25 \%$ en el móvil. En la condición de relajación, el $60 \%$ prefiere disponer de él en el móvil, el $20 \%$ en la TV y un $20 \%$ en su ordenador.

\begin{tabular}{lcc}
\hline & $\begin{array}{c}\text { Condición alegría } \\
\text { Media (DT) }\end{array}$ & $\begin{array}{c}\text { Condición relajación } \\
\text { (Media (DT) }\end{array}$ \\
\hline En qué medida le pareció lógico. & $\bar{X}=7,40(2,51)$ & $\bar{X}=8,40(0,89)$ \\
En qué medida experimentó satisfacción. & $\bar{X}=7,40(3,20)$ & $\bar{X}=9(1,73)$ \\
En qué medida lo recomendaría. & $\bar{X}=7,60(2,79)$ & $\bar{X}=8,80(1,30)$ \\
En qué medida sería útil para otros. & $\bar{X}=7,80(2,38)$ & $\bar{X}=9(1)$ \\
En qué medida es útil en su caso. & $\bar{X}=6,60(2,51)$ & $\bar{X}=8(1,87)$ \\
En qué medida le ha resultado aversivo. & $\bar{X}=2,80(2,58)$ & $\bar{X}=0,80(1,09)$ \\
Utilidad de las imágenes en la inducción emocional (IE). & $\bar{X}=7,80(1,64)$ & $\bar{X}=8,20(0,83)$ \\
Utilidad de las frases en la IE. & $\bar{X}=6,80(1,48)$ & $\bar{X}=6,20(3,11)$
\end{tabular}




\begin{tabular}{lcc}
\hline & $\begin{array}{c}\text { Condición alegría } \\
\text { Media (DT) }\end{array}$ & $\begin{array}{c}\text { Condición relajación } \\
\text { (Media (DT) }\end{array}$ \\
\hline Utilidad de la música en la IE. & $\bar{X}=8,40(1,67)$ & $\bar{X}=9,40(0,89)$ \\
Utilidad del recuerdo autobiográfico en la IE. & $\bar{X}=7,40(2,30)$ & $\bar{X}=8,20(1,09)$ \\
Utilidad del ambiente de RV en la IE. & $\bar{X}=6,40(1,51)$ & $\bar{X}=8,20(1,64)$ \\
Utilidad de la narrativa en la IE. & $\bar{X}=6,80(2,28)$ & $\bar{X}=8,40(1,34)$ \\
\hline
\end{tabular}

\section{Discusión y conclusiones}

En relación con nuestro objetivo de estudio que trata de someter a prueba si la inducción de emociones positivas mediante RV puede modificar la percepción subjetiva de bienestar en personas vulnerables a padecer ansiedad y depresión, podemos decir que los resultados obtenidos no son concluyentes. Estos apoyan parcialmente las hipótesis 1 y 2 , ya que las únicas diferencias significativas encontradas fueron en el nivel de relajación en la condición de relajación y en algunas de las variables de calidad de vida en esta misma condición. Sin embargo, es importante mencionar que sí que se observa una tendencia a incrementar las emociones positivas y disminuir las negativas, tal como refieren estudios previos (Baños y cols., 2004, 2006, 2013). Así mismo se observa una tendencia de cambio positivo después de la inducción emocional en la percepción subjetiva de bienestar en cada una de las variables evaluadas.

En cuanto a los resultados referentes al sentido de presencia y a los efectos secundarios del uso de la RV, podemos decir que ambos confirman parcialmente la hipótesis 3. Por un lado, en el caso del sentido de presencia, en la condición de relajación sí que se observan niveles elevados; no obstante, para la condición de alegría, este es moderado.

Por otro lado, no se identificaron efectos secundarios al uso de la RV en ninguna de las dos condiciones; por lo tanto, se comprueba la hipótesis 4.

La principal limitación de este estudio es su reducida muestra; por lo cual, sería importante replicarlo con una muestra mayor para brindar al estudio de una mayor validez y de resultados más concluyentes. Por otro lado, solo se indujo la emoción en una sola sesión, por lo que sería interesante realizar más sesiones de inducción emocional para observar los posibles cambios que se pueden producir en el tiempo e identificar si estos son significativos.

Finalmente concluir que, debido a la relevancia de la prevención de trastornos como ansiedad y depresión, sería importante seguir investigando los posibles efectos de la inducción de emociones positivas sobre la percepción de calidad de vida y bienestar y su vínculo con la salud mental, así como su potencial utilidad en la prevención de la psicopatología.

\section{Referencias bibliográficas}

Baños, R. M., Botella, C., García-Palacios, A., Villa, H., Perpiñá, C. y Alcañiz, M. (2000). Presence and reality judgment in virtual environments: a unitary construct? Cyberpsychology and Behavior, 3, 327-335.

Baños, R. M., Espinoza, M., García-Palacios, A., Cervera, J. M., Esquerdo, G., Barrajón, E. y Botella, C. (2013). A positive psychological intervention using virtual reality for patients with advanced cancer in a hospital setting: a pilot study to assess feasibility. Supportive Care in Cancer, 21, 263-70. 
Baños, R., Botella, C., Liaño, V., Guerrero, B., Rey, B. y Alcañiz, M. (2004). Sense of presence in emotional virtual environments. Presence: Teleoperators and Virtual Environments, 1 , 156-159.

Baños, R., Liaño, V., Botella, C., Alcañiz, M., Guerrero, B. y Rey, B. (2006). Changing induced moods via virtual reality. Persuasive, 7-15.

Barry, M. M., Clarke, A. M. y Petersen, I. (2015). Promotion of mental health and prevention of mental disorders: priorities for implementation. Eastern Mediterranean Health Journal, $21,503-512$.

Beck, A. T., Ward, C. H., Mendelson, M., Mock, J. y Erbaugh, J. (1961). An inventory for measuring depression. Archives of General Psychiatry 4, 561-571.

Botella, C., Baños, R., García-Soriano, G., Oliver, E., Etchemendy, E., Bretón, J. y Alcañiz, M. (2009). Positive mood induction and well-being. Ponencia presentada en 2nd Conference on Human System Interactions, 517-519.

Bouchard, S., Robillard, G., Renaud, P. y Bernier, F. (2011). Exploring new dimensions in the assessment of virtual reality induced side effects. Journal of Computer and Information Technology, 1, 20-32.

First, M. B., Gibbon, M., Spitzer, R. L., Williams, J. B. y Benjamin L. S. (1997). Structured Clinical Interview for DSM-IV Axis II Personality Disorders, (SCID-II). Washington, D.C.: American Psychiatric Press.

Folkman, S. y Moskowitz, J. T. (2000). Positive affect and the other side of coping. American Psychologist, 55, 647-654.

Fredrickson, B. L. (1998). What good are positive emotions? Review of General Psychology, 2, 300-319.

Gross, J. J. y Levenson, R. W. (1995). Emotion elicitation using films. Cognition and Emotion, 9, 87-108.

Kennedy, R. S., Lane, N. E., Berbaum, K. S. y Lilienthal, M. G. (1993). Simulator Sickness Questionnaire: an enhanced method for quantifying simulator sickness. International Journal of Aviation Psychology, 3, 203-220.

Livingstone, K. M. y Srivastava, S. (2012). Up-regulating positive emotions in everyday life: Strategies, individual differences, and associations with positive emotion and well-being. Journal of Research in Personality, 46, 504-516.

Mezzich, J. E., Ruiperez, M. A., Perez, C., Yoon, G., Liu, J. y Mahmud, S. (2000). The Spanish version of the Quality of Life Index: presentation and validation. Journal of Nervous and Mental Disease, 188, 301-305.

Organización Mundial de la Salud. (2011). Carga mundial de trastornos mentales y necesidad de que el sector de la salud y el sector social respondan de modo integral y coordinado a escala de país (Informe de la Secretaría). Consejo Ejecutivo, 1-6.

Peterson, R. A. y Reiss, R. J. (1992). Anxiety Sensitivity Index Manual (2. ${ }^{a}$ ed.). Worthington, $\mathrm{OH}$ : International Diagnostic Systems.

Sandín, B., Chorot, P., Lastao, L., Joiner, T .E., Santed, M. A. y Valiente, R. M. (1999). Escalas PANAS de afecto positivo y negativo: validación factorial y convergencia transcultural. Psicothema, 11, 37-51.

Sandín, B., Valiente, R. M., Chorot, P. y Santed, M. A. (2007). ASI-3: Nueva escala para la evaluación de la sensibilidad a la ansiedad. Revista de Psicopatología y Psicología Clínica, 12, 91-104.

Santos, V., Paes, F., Pereira, V., Arias-Carrión, O., Cardoso, A., Carta, M. G., Nardi, A. E. y Machado, S. (2013). The role of positive emotion and contributions of positive psychology in depression treatment: systematic review. Clinical Practice \& Epidemiology in Mental Health, 9, 221-237. 
Sanz, J. y Vázquez, C. (1998) Fiabilidad, validez y datos normativos del inventario para la depresión de Beck. Psicothema, 10, 303-318.

Spielberger, C. D., Gorsuch, R. L. y Lushene, R. E. (1970). Manual for the State-Trait Anxiety Inventory. Palo Alto, CA: Consulting Psychologists Press.

Watson, D., Clark, L. A. y Tellegen, A. (1988). Development and validation of brief measures of positive and negative affect: the PANAS scales. Journal of Personality and Social Psychology, 54, 1063-1070.

Wood, A. M. y Tarrier, N. (2010). Positive clinical psychology: a new vision and strategy for integrated research and practice. Clinical Psychology Review, 30, 819-829. 